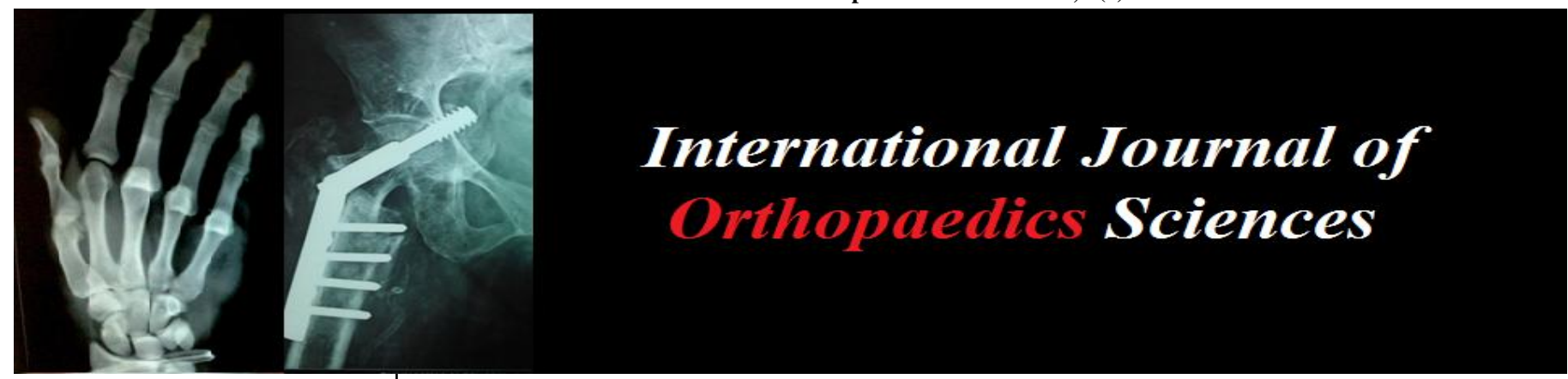

ISSN: $2395-1958$

IJOS 2018; 4(1): 69-76

(C) 2018 IJOS

www.orthopaper.com

Received: 01-11-2017

Accepted: 02-12-2017

Ravikant Jain

HOD and Prof, Dept of orthopaedics, SAMC \& PGI,

Indore, Madhya Pradesh, India

Aamir Johar

Junior Resident, Dept of

orthopaedics, SAMC \& PGI,

Indore, Madhya Pradesh, India

Correspondence

Ravikant Jain

HOD and Prof, Dept of

orthopaedics, SAMC \& PGI,

Indore, Madhya Pradesh, India

\section{To evaluate the role of high resolution ultrasonography in diagnosis of knee osteoarthritis}

\author{
Ravikant Jain and Aamir Johar
}

DOI: https://doi.org/10.22271/ortho.2018.v4.i1b.11

\section{Abstract}

Introduction: Osteoarthritis $(\mathrm{OA})$ is a progressive and disabling joint disease. It is one of the most frequently occurring health problems for middle-aged and older people. ${ }^{[1,2]} \mathrm{OA}$ can occur in every synovial joint, but is most common in hip, knee, hand, foot and spine. ${ }^{[3]}$ It is characterized by joint pain and limited function of the joint. In a synovial joint several structures can cause these clinical symptoms. The Kellgren and Lawrence $(\mathrm{K} \& \mathrm{~L})$ classification criteria are the most widely used radiographic classification criteria to identify and grade OA.

Objectives: To evaluate the role of High Resolution Ultrasonography in knee osteoarthritis.

Material and Methods: Fifty patients attending the outpatient orthopaedics department of our institute with unilateral or bilateral knee pain were included. 32 patients had bilateral symptomatic knee OA and 18 patients had unilateral symptomatic OA. All knees were subjected to radiographic examination and HRUS assessment.

Conclusion: HRUS plays a significant role in early diagnosis of knee osteoarthritis, as well as its progression and therapy monitoring. It is desirable as a quick and easy method of screening for articular cartilage abnormalities and extra-articular abnormalities. Its cost effectiveness, dynamic scanning capabilities and therapeutic guidance providing abilities make it valuable in all settings.

Keywords: Osteoarthritis, HRUS, K\&L Classification

\section{Introduction}

Osteoarthritis (OA) is a progressive and disabling joint disease. It is one of the most frequently occurring health problems for middle-aged and older people. ${ }^{[1,2]}$ OA can occur in every synovial joint, but is most common in hip, knee, hand, foot and spine. ${ }^{[3]}$ It is characterized by joint pain and limited function of the joint. In a synovial joint several structures can cause these clinical symptoms. Bone, cartilage, synovial fluid, ligaments and also the muscles around the joint are tissues that change with OA and affect the function of the joint. ${ }^{[4]}$ Several tissues might be a starting point for pathways that lead to OA. Cartilage might be a tissue in which the patho-physiological process of OA starts, but biochemical and imaging studies have shown that synovium and bone can also be starting points.

A diagnosis of OA is mainly based on symptoms. Recommendations for the diagnosis of knee OA were published in 2010. ${ }^{[5]}$ They include three main symptoms: knee pain, short-lived morning stiffness, and functional limitation in combination with three signs on physical examination (crepitus, restricted movement and bony enlargement).

In all joints, the prevalence of $\mathrm{OA}$ increases with age. In addition, the elderly population continues to increase, resulting in an even higher clinical and economic burden of OA. ${ }^{[6]}$ Worldwide estimates are that $9.6 \%$ of men and $18.0 \%$ of women aged 60 years and over have symptomatic OA. For radiological knee OA these estimates are somewhat higher, even at a younger age (45 years and over): $14.1 \%$ for men and $22.8 \%$ for women. ${ }^{[2]}$

When a patient presents in healthcare with symptoms of OA, it is likely that there is already pathological damage in the knee, perhaps for a long period. Symptomatic treatment (e.g. exercise and mechanical interventions, like insoles) might be more effective at an earlier clinical stage; the same probably applies to disease-modifying treatment. In a pre-clinical stage with early pathological damage of the knee joint, such disease-modifying treatment might be even more promising. 
Radiographs have for long been the most frequently used and the standard images for visualizing OA. But as mentioned above there is lack of agreement between clinical and radiographic knee OA. And also it is not useful in predicting preclinical OA.

MRI has the advantage of direct assessment of the cartilage, which is still thought to be an important tissue in OA, as well as the advantage of visualizing all the other important tissues of the joint. MRI has another important potential advantage compared to radiographs: it can show structural damage or lesions earlier than can be seen clinically or on radiographs. Therefore, identifying OA features at a pre-clinical stage with MRI makes it to be a test of choice. But it is not cost effective.

Knee high resolution ultrsonography (HRUS) is desirable as a quick and easy method of screening for articular cartilage abnormalities and extra-articular abnormalities. Its cost effectiveness, dynamic scanning capabilities and therapeutic guidance providing abilities make it useful in our settings. In addition, for some patients sonography may be the test of choice, because of the presence of a pacemaker or other absolute or relative contraindication to MRI.

By this study we want to evaluate role of HRUS in knee osteoarthritis as a early diagnostic tool.

\section{Aims \& objectives}

* To evaluate the role of High Resolution Ultrasonography in knee osteoarthritis.

\section{Materials \& methods}

The present study, "To Evaluate the Role of High Resolution Ultrasonography in Diagnosis of Knee Osteoarthritis" was carried out in the Department of Orthopaedics, Sri Aurobindo Medical College \& P.G. Institute, Indore from $1^{\text {st }}$ January, 2016 to $30^{\text {th }}$ September, 2017 after approval from institutional research and ethical committee with main objective to evaluate the role of High Resolution Ultrasonography (HRUS) in knee osteoarthritis.

Study population will comprise of patients above 40 years of age.

The exclusion criteria include all patients with history of recent trauma, mechanical knee derangement, fibromyalgia, inflammatory arthritis, microcrystalline arthropathy, recent surgery and known cases of malignancies around knee.

Fifty patients attending the outpatient orthopaedics department of our institute with unilateral or bilateral knee pain were included. No patient has received arthrocentesis and/or intraarticular steroid injection during the last 3 months. 32 patients had bilateral symptomatic knee OA and 18 patients had unilateral symptomatic OA. Thus total of 82 symptomatic knees and 18 asymptomatic knees constituted part of our study. All symptomatic and asymptomatic knees were subjected to radiographic examination and HRUS assessment. Weight-bearing anteroposterior (AP) and lateral knee radiographs were read by radiologist blinded to the clinical and US findings who assessed the severity of OA on the AP view using the Kellgren and Lawrence (K-L) scale (scores 0-4) and for the presence of patello-femoral (PF) degenerative signs.

A written informed consent was obtained from all patients prior to the clinical, US and radiographic evaluation.

An HRUS examination of both the knees was performed on Philips iU22 machine using a high frequency linear probe of 5-12 MHz.US examination was performed according to standardized scanning method
Findings were recorded on a proforma for the study and statistical was analysis done.

Articular cartilage of the femur is clearly depicted by sonography. The synovial membrane is carefully studied by sonography. In fact, it is possible to measure its thickness and show the presence of villi and septa. In this way the episodic synovitis that may appear in OA is easily detected and useful information is obtained for disease and therapy monitoring. In a normal knee its thickness is between 2 and $3 \mathrm{~mm}$. In an osteoarthritic joint the synovial membrane is usually thickened when synovitis is present

Synovial fluid is very well evaluated, sonography may detect the quantity of this fluid, revealing the presence of joint effusion with very high sensitivity. [7] The physiological amount of synovial fluid in a normal knee is between 3 and $3.5 \mathrm{ml}$. Hydrarthrosis is demonstrated by the evidence of anechoic or hypoechoic collection within the joint cavity.

It is possible to evaluate the regularity of the bone surfaces of the femur and the tibia. Osteophytes are easily detected, their presence being demonstrated by the finding of irregularity at the joint margins.

Another frequent alteration in $\mathrm{OA}$ is capsular fibrosis. ${ }^{[8]}$ Sonography shows the thickening of the capsule.

Evaluation of the calf may show the presence of Baker's cysts. They are frequently found in OA, especially when effusion is present and the gastrocnemius-semimembranosus bursa communicates with the joint cavity.

Classification Criteria: The Kellgren and Lawrence (K\&L) classification criteria are the most widely used radiographic classification criteria to identify and grade OA

\section{Review of literature}

Keen et al. (2009) did a study to undertake a systematic review of the published literature evaluating ultrasonography as an assessment tool in osteoarthritis. Results: Forty-seven studies were identified that utilized ultrasonography to assess structural pathology in osteoarthritis. Doppler function was only assessed in 10 studies and contrast agents in one. There was heterogeneity with regard to the pathology examined, the definition of pathology, quantification and the reporting of these factors. There was also a lack of construct and criterion validity and data demonstrating reliability and sensitivity to change. Whereas there is increasing evidence of the validity of ultrasonography in detecting structural pathology in inflammatory arthritis, more work is required to develop standardized definitions of pathology and to demonstrate the validity of ultrasonography in osteoarthritis. [9]

Study was conducted by Abraham et al. (2011) to measure the inter-rater reliability and validity of ultrasound imaging in the detection of features of knee OA and found that Reliability for osteophyte presence was 0.77 (right femur), 0.65 (left femur) and 0.88 for both tibia. ICCs for effusion size were 0.70 (right) and 0.85 (left). Moderate to substantial agreement was found in cartilage thickness measurements. And Validity for osteophytes, was moderate to excellent at 0.52(right) and 0.75(left). Concluded that excellent agreement was found between ultrasound observers for the presence of osteophytes and measurement of effusion size; it was moderate to substantial for femoral cartilage thickness. Moderate to substantial agreement was observed between ultrasound and radiographs for osteophyte presence. ${ }^{[10]}$

Kazam et al. (2011) did a sonographic evaluation of femoral trochlear cartilage in patients with knee pain. The purpose of this study was to investigate whether routine clinical sonographic evaluation of femoral trochlear cartilage can 
identify abnormalities in patients with knee pain. Using repeated measures analysis of variance, there were significant differences $(\mathrm{P}=.02)$ for both decreased cartilage clarity $(\mathrm{df}=1 / 28 ; \mathrm{F}=5.76)$ and increased grade $(\mathrm{df}=1 / 28 ; \mathrm{F}=5.77)$ in patients. There was also a non-significant $(P>.05)$ trend toward more frequent calcifications, osteophytes, and bony irregularity in patients. Routine clinical sonography can identify femoral trochlear cartilage abnormalities in patients with knee pain and therefore can be a useful adjunct to other imaging tests for identifying arthritic changes in the knee. ${ }^{[1]}$ Hammer et al. (2016) did a study to assess whether ultrasonography (US) is reliable for the evaluation of inflammatory and structural abnormalities in patients with knee osteoarthritis (OA). Intra- and interobserver reliability scores were moderate to good for synovitis (mean $\kappa 0.67$ and 0.52 , respectively) as well as moderate to good for the global synovitis ( 0.70 and 0.50 , respectively). Mean intra- and interobserver reliability $\kappa$ for cartilage damage, medial meniscal damage and osteophytes ranged from fair to good (0.55 and 0.34, 0.75 and 0.56, 0.73 and 0.60, respectively).Using a standardized protocol, dichotomous and semi quantitative US scoring of pathological changes in knee OA can be reliable. ${ }^{[12]}$

\section{Observations and Results}

There were $6(12 \%)$ in the age group $40-50$ years, $24(48 \%)$ were in the age group 51-60 years, $13(26 \%)$ were in the age group 61-70 years and 7 (14\%) were in the age group 71-80 years.

There were $35(70 \%)$ females and $15(30 \%)$ males in the study. There was a female preponderance in the study.

Table 1: Distribution of patients according to BMI grading $(\mathrm{N}=50)$

\begin{tabular}{|c|c|c|c|}
\hline S. No. & BMI Grading & No. & \% \\
\hline 1. & Underweight $\left(<18.5 \mathrm{~kg} / \mathrm{m}^{2}\right)$ & 4 & 8.0 \\
\hline 2. & Normal $\left(18.50-24.99 \mathrm{~kg} / \mathrm{m}^{2}\right)$ & 6 & 12.0 \\
\hline 3. & Overweight $\left(\geq 25.00-29.99 \mathrm{~kg} / \mathrm{m}^{2}\right)$ & 33 & 66.0 \\
\hline 4. & Obese $\left(\geq 30.00 \mathrm{~kg} / \mathrm{m}^{2}\right)$ & 7 & 14.0 \\
\hline & Total & 50 & 100.0 \\
\hline
\end{tabular}

The above table shows the distribution of patients according to BMI grading. There were $4(8 \%)$ patients who were underweight, $6(12 \%)$ patients who were normal, $33(66 \%)$ patients were overweight and $7(14 \%)$ patients were obese. Majority of the patients were overweight.

Table 2: Distribution of patients according to laterality

$(\mathrm{N}=50)$
\begin{tabular}{|c|c|c|c|}
\hline S. No. & Laterality of involvement & No. & $\%$ \\
\hline 1. & Unilateral & 18 & 36.0 \\
\hline 2. & Bilateral & 32 & 64.0 \\
\hline & Total & 50 & 100.0 \\
\hline
\end{tabular}

The above table shows the distribution of patients according to laterality. $18(36 \%)$ of the patients had shown unilateral involvement and $32(64 \%)$ of the patients had shown bilateral involvement.

Table 3: Distribution of patients according to presenting symptoms $(\mathrm{N}=50)$

\begin{tabular}{|c|c|c|c|}
\hline S. No. & Presenting Symptoms & No. & \% \\
\hline 1. & Pain on walking & 40 & 80.0 \\
\hline 2. & Pain on stair climbing & 20 & 40.0 \\
\hline 3. & Pain on lying & 35 & 70.0 \\
\hline 4. & Pain on sitting & 6 & 12.0 \\
\hline 5. & Pain on standing & 4 & 8.0 \\
\hline
\end{tabular}

The above table shows the distribution of patients according to presenting symptoms. The most common presenting symptom was pain on walking $40(80 \%)$, followed by pain on lying in $35(70 \%)$ of the patients, $20(40 \%)$ patients had pain on stair climbing, $6(12 \%)$ patients had pain on sitting and 4 $(8 \%)$ of the patients had pain on standing.

Table 8, Fig 24: X-ray Findings in Patients

\begin{tabular}{|c|c|c|c|c|c|}
\hline \multirow{2}{*}{$\begin{array}{l}\text { S. } \\
\text { No. }\end{array}$} & \multirow[t]{2}{*}{ X-ray Findings } & \multicolumn{2}{|c|}{$\begin{array}{c}\text { Symptomatic } \\
(\mathrm{n}=82)\end{array}$} & \multicolumn{2}{|c|}{$\begin{array}{c}\text { Asymptomatic } \\
(\mathrm{n}=18)\end{array}$} \\
\hline & & No. & $\%$ & No. & $\%$ \\
\hline 1. & Joint space reduction & 28 & 34.1 & 10 & 55.6 \\
\hline 2. & Osteophytes formation & 20 & 24.4 & 5 & 27.8 \\
\hline 3. & Subarticular sclerosis & 14 & 17.1 & 4 & 22.2 \\
\hline 4. & Geode formation & 6 & 7.3 & 2 & 11.1 \\
\hline 5. & Loose bodies & 8 & 9.8 & 2 & 11.1 \\
\hline 6. & Deformity of both ends & 6 & 7.3 & 0 & 0.0 \\
\hline 7. & Normal & 0 & 0.0 & 6 & 33.3 \\
\hline
\end{tabular}

The above table shows the distribution of knees according to $\mathrm{X}$-ray findings. There were 82 symptomatic and 18 asymptomatic knees of the 50 patients. In symptomatic knees, $28(34.1 \%)$ knees had joint space reduction, 20 (24.4\%) knees had osteophytes formation, $14(17.1 \%)$ knees had subarticular sclerosis, $6(7.3 \%), 6(7.3 \%)$ knees had Geode formation, 8 $(9.8 \%)$ knees had loose bodies, $6(7.3 \%)$ knees had deformity of the both ends as seen on the x-ray.

In the asymptomatic knees, 10 (55.6\%) knees had joint space reduction, $5(27.8 \%)$ knees had osteophytes formation, 4 $(22.2 \%)$ knees had subarticular sclerosis, $2(11.1 \%)$ knees had Geode formation, $2(11.1 \%)$ knees had loose bodies and 6 $(33.3 \%)$ knees had shown normal findings on x-ray.

Joint space reduction, osteophytes formation and subarticular sclerosis were the commonest findings on $\mathrm{x}$-ray in both the symptomatic as well as asymptomatic knees. 


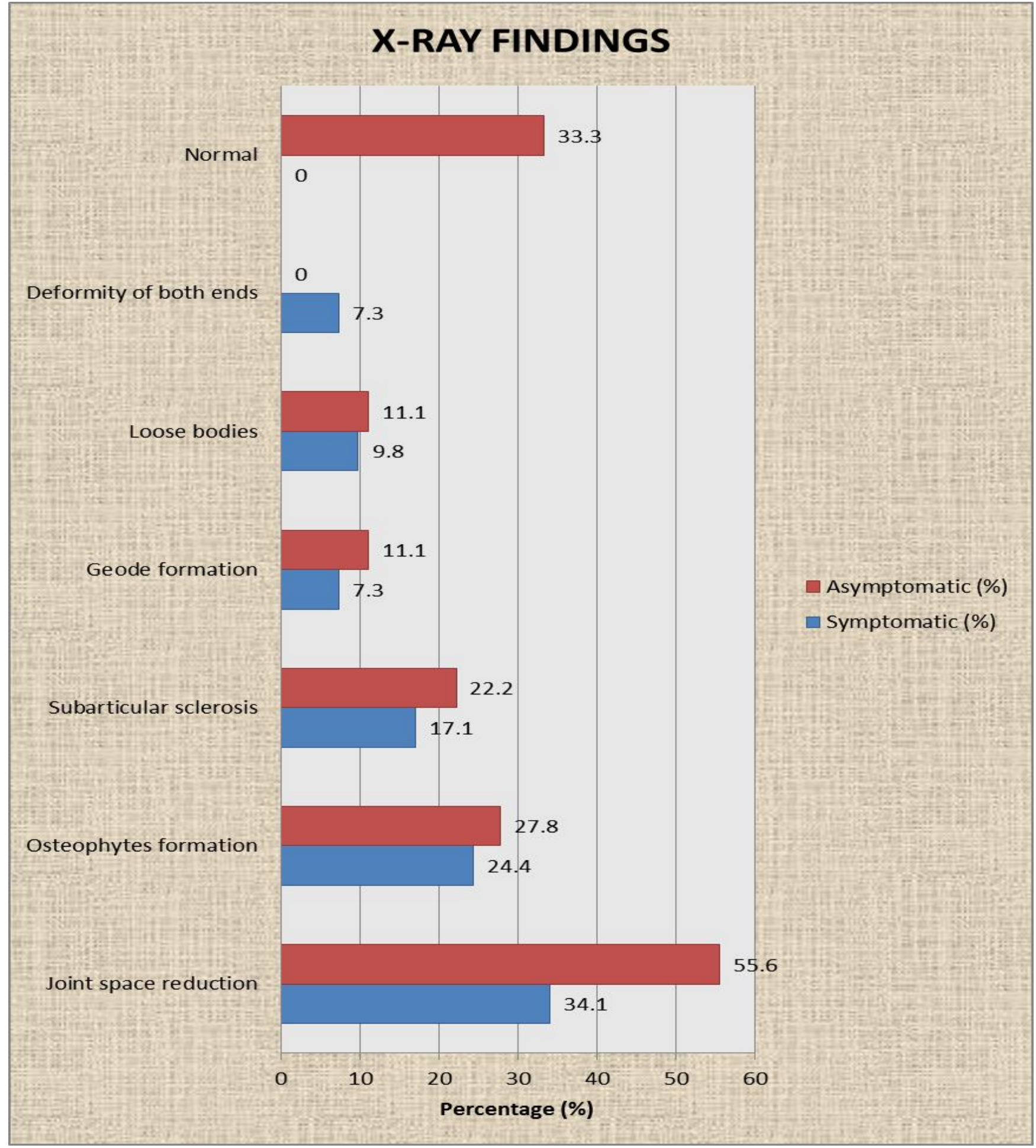

Fig 24

Table 9: K \& L Grading

$(\mathrm{N}=100)$

\begin{tabular}{|c|c|c|c|c|c|}
\hline \multirow{2}{*}{ S. No. } & \multirow{2}{*}{ K\&L Grading } & \multicolumn{2}{|c|}{$\begin{array}{c}\text { Symptomatic } \\
(\mathbf{n}=\mathbf{8 2})\end{array}$} & \multicolumn{2}{c|}{$\begin{array}{c}\text { Asymptomatic } \\
(\mathbf{n = 1 8})\end{array}$} \\
\cline { 3 - 6 } & & $\mathbf{N o .}$ & $\mathbf{\%}$ & No. & \% \\
\hline 1. & Grade I & 28 & 21.0 & 10 & 73.7 \\
\hline 2. & Grade II & 21 & 30.6 & 7 & 18.4 \\
\hline 3. & Grade III & 18 & 24.2 & 1 & 7.9 \\
\hline 4. & Grade IV & 15 & 24.2 & 0 & 0.0 \\
\hline & Total & 82 & 100.0 & 18 & 100.0 \\
\hline
\end{tabular}

The above table shows the distribution of knees according to various K \& L Grading.
In the symptomatic knees, 28 (21.0\%) knees had Grade I, 21 $(30.6 \%)$ had Grade II, 18 (24.2\%) had Grade III and 15 (24.2\%) had Grade IV K\&L Grading.

In the asymptomatic knees, 10 (73.7\%) knees had Grade I, 7 (18.4\%) had Grade II, 1 (7.9\%) had Grade III K\&L Grading, while there were none in Grade IV.

In the asymptomatic group, the distribution of knees was nearly similar in all the grades, while in the asymptomatic knees, majority of the knees were in the Grade I K\&L grading. 


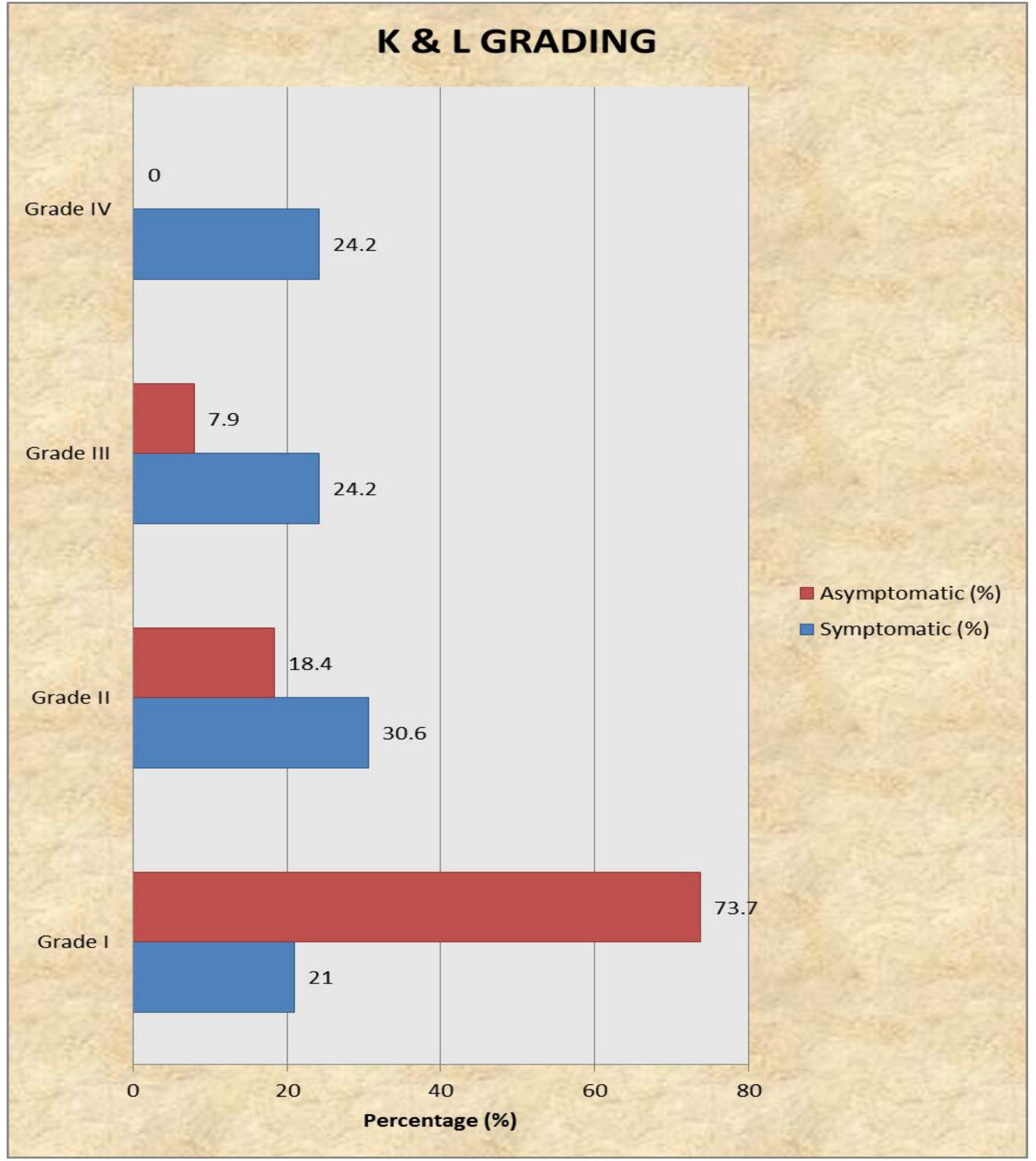

Fig 25: K \& L Grading

Table No. 10, Fig. 26: Ultrasonographic Findings in Knees

\begin{tabular}{|c|c|c|c|c|c|}
\hline \multirow{2}{*}{ S. No. } & \multirow{2}{*}{ Ultrasonographic Findings } & \multicolumn{2}{|c|}{$\begin{array}{c}\text { Symptomatic } \\
(\mathrm{n}=82)\end{array}$} & \multicolumn{2}{|c|}{$\begin{array}{c}\text { Asymptomatic } \\
(n=18)\end{array}$} \\
\hline & & No. & $\%$ & No. & $\%$ \\
\hline 1. & Articular cartilage thinning & 80 & 97.6 & 10 & 55.6 \\
\hline 2. & Loss of cartilage interface & 78 & 95.1 & 8 & 44.4 \\
\hline 3. & Osteophyte formation & 75 & 91.5 & 6 & 33.3 \\
\hline 4. & Medial meniscal protrusion & 75 & 91.5 & 4 & 22.2 \\
\hline 5. & Lateral meniscal protrusion & 70 & 85.4 & 3 & 16.7 \\
\hline 6. & Joint effusion & 20 & 24.4 & 1 & 5.6 \\
\hline 7. & Popliteal cyst & 15 & 18.3 & 0 & 0.0 \\
\hline
\end{tabular}

The above table shows the distribution according to ultrasonographic findings.
In the asymptomatic group, $80(97.6 \%)$ knees had shown articular cartilage thinning, $78(95.1 \%)$ had shown loss of cartilage interface, $75(91.5 \%)$ had shown osteophyte formation, 75 (91.5\%) had shown medial meniscal protrusion, $70(85.4 \%)$ had shown lateral meniscal protrusion, $20(24.4 \%)$ had shown joint effusion and 15 (18.3\%) had shown popliteal cyst on ultrasonography.

In the symptomatic group, $10(55.6 \%)$ had shown articular cartilage thinning, $8(44.4 \%)$ had shown loss of cartilage interface, $6(33.3 \%)$ had shown osteophyte formation, 4 (22.2\%) had shown medial meniscal protrusion, 3 (16.7\%) had shown lateral meniscal protrusion, $1(5.6 \%)$ had shown joint effusion and one had shown popliteal cyst on ultrasonography. 


\section{ULTRASONOGRAPHIC FINDINGS}

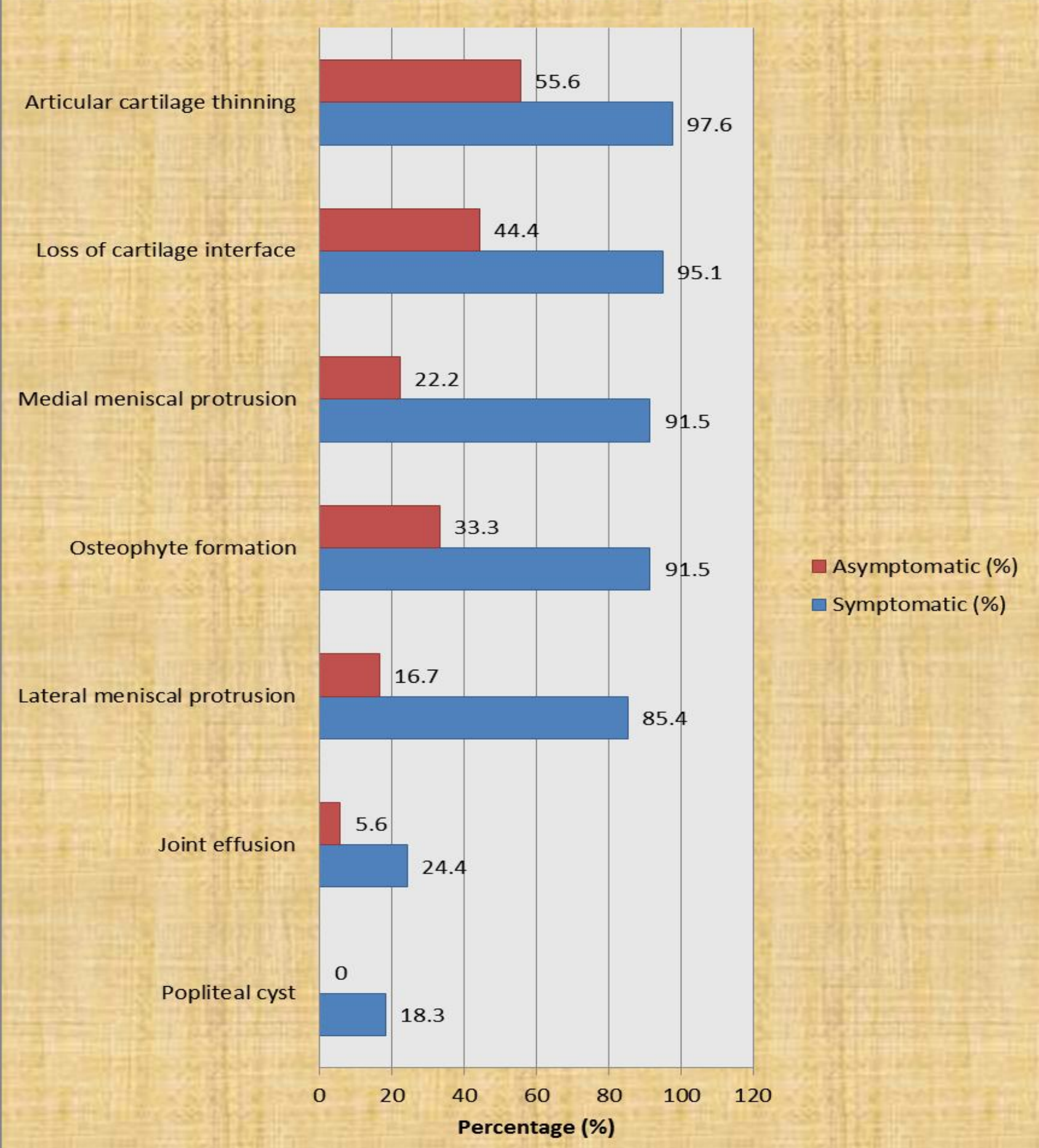

Fig 26

\section{Discussion}

*Osteoarthritis (OA) is one of the most common medical conditions in elderly people. [1] Worldwide estimates suggested that $9.6 \%$ of men and $18.0 \%$ of women aged 60 years have symptomatic OA of the hips or knees. ${ }^{[2]}$ It is also the most common reason for restricted daily activity ${ }^{[3]}$ with significantly impact on the quality of life among affected people. ${ }^{[4]}$ Pain is the predominant symptom of OA knee and is the main reason for medical consultation; besides, it the main reason of disability especially during painful episodes. ${ }^{[5-7]}$

\section{Age Group}

In the present study, majority of the patients were in the age group 51-60 years (48\%), followed by $61-70$ years (26\%) and $14 \%$ in the age group $71-80$ years.

\section{Gender}

In our study, female $(70 \%)$ preponderance was seen in comparison to male $(30 \%)$.

\section{Body Mass Index (BMI)}

Majority of the patients in our study were overweight (66\%), followed by $14 \%$ obese and $12 \%$ normal, while $8 \%$ of the patients were underweight.

In our study, the higher the KL grading was associated with more pain in general. The finding is in line with a recent 
systematic review that higher grade of osteoarthritis (K/L 3 or above) is a stronger predictor of pain than lower grades $(\mathrm{K} / \mathrm{L}$ 2 or less). ${ }^{[8]}$

Synovitis is a sign of joint inflammation and its association with pain when sitting and not with walking or stair climbing suggests that the resting OA knee pain during sitting is more likely inflammatory pain.

This study showed that knee effusion has positive correlation with symptoms upon walking and climbing, but not with sitting. Similar findings are found in other overseas studies. $[13,14]$ This suggests that the knee effusion among OA knee patients relates more to mechanical rather than inflammatory pain. Knee effusion has been shown to affect knee mechanics and muscle activity during gait in individuals with knee osteoarthritis and therefore can be a cause of the mechanical pain by itself. ${ }^{[15]}$

The knee joint is a complex joint consisting of a patellofemoral joint (PFJ) articulating the patella with the femoral condyle and two tibiofemoral joints, one medial and one lateral (medial and lateral TFJs), articulating the medial and lateral tibial plateau with the corresponding femoral condyles. Although X-rays measure mainly bony pathologies and ultrasound on soft tissue pathologies, both the X-ray and ultrasound findings in our study showed two trends in common (1) walking pain is related to pathologies of the either the lateral or medial TFJ; (2) stair climbing pain is related to both TFJ pathologies and also to the PFJ pathology. Meniscus subluxation was highly associated with painful OA knee. ${ }^{[26]}$ Our HRUS studies showed that lateral meniscal extrusion was associated with increased pain on walking. Xray studies, on the other hand, showed pain on walking was associated with the narrowing of the medial TFJ at mid-point. When stair climbing, pathologies from both the medial and lateral TFJ are implicated. The US findings showed that stair climbing pain has a positive correlation to both medial meniscal protrusion and lateral meniscus protrusion; whereas on X-rays, the narrower the medial TFJ at midpoint and the wider the lateral TFJ at midpoint, more the pain. Both of these X-rays findings are commonly found among bow knees (Genu Varus) which are commonly found among OA knee patients.

Pain with stair climbing, especially on descending stairs is a well-known feature with OA knee involving the PFJs. ${ }^{[16]}$ Our $\mathrm{X}$-ray studies found that the narrower the lateral facet joint, the more the pain on stair climbing. However, our US finding showed that the thickness of the lateral femoral condyle cartilage, which is a measurement of the lateral PFJ, has no association with stair climbing. Such finding is expected because ultrasound can only measure, through the sky view, the cartilage of the upper part of the femoral condyle.

Some limitations of our study should be mentioned. Firstly, we used the conventional standing AP knee radiograph whereas a semi flexion AP view would be more accurate for studying the FT space. Moreover, we did not use an axial view for evaluating the PF space. Nevertheless, we performed the routine views mostly used in daily clinical practice for radiologically assessing severity and progression of knee OA. Secondly, the US inter-observer reliability was not studied. HRUS has been viewed as one of the most operatordependent imaging technique. This fact is partly due to the intrinsic real time nature of US images acquisition. However, in our study all the HRUS examinations were performed by the same radiologist who has 9 years of experience in MSK US. In addition, the recent study by Scheel et al. has revealed an excellent inter-observer reliability (kappa value of 1) between 14 experts in MSK US for the US detection of knee pathological findings including effusion, bone surface abnormalities, tendon lesions, bursitis and Baker's cyst. In the same study, the overall sensitivity and specificity of US compared with MRI for diagnosing knee abnormalities were $90.5 \%$ and $87.5 \%$, respectively.

In Indian population there is habit of squatting and sitting cross legged, which leads to more incidence of knee OA than Hip OA.

\section{Conclusion}

Knee sonography is desirable as a quick and easy method of screening for articular cartilage abnormalities and extraarticular abnormalities. Its cost effectiveness, dynamic scanning capabilities and therapeutic guidance providing abilities make it useful in our settings. In addition, for some patients sonography may be the test of choice, because of the presence of a pacemaker or other absolute or relative contraindication to MRI. High resolution ultra-sonography can identify femoral trochlear cartilage abnormalities in patients with knee pain and therefore can be a useful adjunct to other imaging tests for identifying arthritic changes in the knee this diagnostic imaging method makes it possible to evaluate most of the structures involved in this disease, carefully revealing the possible changes produced. This method assures the repeatability of the technique so that both the disease and therapy monitoring is possible., sonography may detect the quantity of synovial fluid, revealing the presence of joint effusion with very high sensitivity. Hence, HRUS plays a significant role in early diagnosis of knee osteoarthritis, as well as its progression and therapy monitoring.

\section{References}

1. Buckwalter JA, Martin JA. Osteoarthritis. Adv Drug Deliv Rev. 2006; 58:150-67.

2. Woolf AD, P\$eger B. Burden of major musculoskeletal conditions. Bull World Health Organ. 2003; 81:646-56.

3. Buckwalter JA, Saltzman C, Brown T. The impact of osteoarthritis: implications for research. ClinOrthopRelat Res. 2004; (427 Suppl):S6-15.

4. Andriacchi TP, Koo S, Scanlan SF. Gait mechanics in\$uence healthy cartilage morphology andosteoarthritis of the knee. J Bone Joint Surg Am. 2009; 91Suppl 1:95101.

5. Samuels J, Krasnokutsky S, Abramson SB. Osteoarthritis: a tale of three tissues. Bull NYU HospJt Dis. 2008; 66: 244-50.

6. Zhang W, Doherty M, Peat G, Bierma-Zeinstra MA, Arden NK, Bresnihan B, et al. EULAR evidence-based recommendations for the diagnosis of knee osteoarthritis. Ann Rheum Dis. 2010; 69:483-9.

7. Hunter DJ. Pharmacologic therapy for osteoarthritis--the era of disease modification. Nat Rev Rheumatol. 2011; 7:13-22.

8. Reginster JY. The prevalence and burden of arthritis. Rheumatology (Oxford). 2002; 41Supp 1:3-6.

9. Keen HI, Wakefield RJ, Conaghan PG. A systematic review of ultrasonography in osteoarthritis Ann Rheum Dis 2009; 68:611-9.

10. Abraham. Reliability and validity of ultrasound imaging of features of knee osteoarthritis in the community BMC Musculoskeletal Disorders 2011; 12:70.

11. Kazam JK, Nazarian LN, Miller TT, Sofka CM, Parker L, Adler RS. 
12. Hammer HB, Bruyn GA, Naredo E, Damjanov $\mathrm{N}$ on behalf of the Ultrasound Task Force, et alAn OMERACT reliability exercise of inflammatory and structural abnormalities in patients with knee osteoarthritis using ultrasound assessmentAnnals of the Rheumatic Diseases 2016; 75:842-846.

13. Kellgren JH, Lawrence JS. Radiological assessment of osteo-arthrosis. Ann Rheum Dis 1957; 16:494-502.

14. Lawrence JS. Rheumatism in populations. London, UK: William Heinemann Medica Books Ltd, 1977, 98-155.

15. Kellgren JH, Jeffrey MR, Ball J. The epidemiology of chronic rheumatism. Atlas of standard radiographsof arthritis. Oxford, UK: Blackwell Scientific Publications, 1963, 7-11.

16. Neustadt D, Caldwell J, Bell M. Clinical Effects of Intraarticular Injection of High Molecular Weight Hyaluronan (Orthovisc) in osteoarthritis of the knee: a randomized, controlled, multicenter trial. J Rheumatol $2005 ; 32: 1928-36$.

17. Kellgren JH, Lawrence JS. Radiological assessment of osteoarthrosis. Ann Rheum Dis. 1957; 16:494-502. 